\title{
THE EFFECT OF REINSURANCE ON THE DEGREE OF RISK ASSOCIATED WITH AN INSURER'S PORTFOLIO
}

\author{
M. Andreadakis and H. R. Waters \\ Heriot-Watt University, Edinburgh
}

\section{INTRODUCTION}

There are many reasons why an insurer may choose to reinsure a part of his portfolio (see, for example, CARTER (1979, p. 5 ff.)) and many ways in which he can assess the effectiveness of the reinsurance arrangements he makes. In this paper we assume the insurer wishes to reinsure a part of his portfolio in order to reduce its "riskiness". We take as given a portfolio consisting of $n$ independent risks together with the total premium charged to insure these risks and we investigate the effect on the degree of risk associated with the portfolio (see $\$ 3$ for a definition) of varying the excess of loss or proportional reinsurance limits for each risk.

\section{ASSUMPTIONS AND NOTATION}

We are given an insurance portfolio consisting of $n$ independent risks. A risk may consist of a single policy or a group of policies: the essential points being that a reinsurance limit, either excess of loss or proportional, is the same for all claims arising from a particular risk, although reinsurance limits may vary from one risk to another. We assume the claims arising from each risk have a compound Poisson distribution. To be more precise, we assume the number of claims arising from the $i$-th risk is a Poisson process with mean $p_{i}$ claims each year and the size of each claim has distribution function $F_{i}$. As usual, the size of a claim is independent of the time at which it occurs and of all other claims. We also assume that $F_{i}(\mathrm{o})=\mathrm{o}$ for each $i$, so that we consider only positive claims amounts. We take as given the total annual premium, $P$, charged by the insurer in respect of these risks. We make no assumption about the way in which $P$ is calculated but we do assume that

$$
P>\sum_{i=1}^{n} p_{i} \int_{0}^{\infty} x d F_{i}(x)
$$

We now turn our attention to the reinsurance of the portfolio and to avoid any possibility of confusion we state precisely what we mean by excess of loss and proportional reinsurance. Suppose a single claim for an amount $X$ arises from the $i$-th risk. If the insurer has arranged excess of loss reinsurance for 
the portfolio with reinsurance limits specified by $\left(M_{1}, M_{2}, \ldots, M_{n}\right)$ then the insurer pays

$$
X \text { if } X \leqslant M_{i} \quad \text { or } \quad M_{i} \text { if } X>M_{i}
$$

and the reinsurer pays the excess amount, if any. If the insurer has arranged proportional reinsurance with reinsurance limits specified by $\left(a_{1}, a_{2}, \ldots, a_{n}\right)$ then the insurer pays $a_{i} X$ and the reinsurer pays $\left(1-a_{i}\right) X$. The symbols $M_{i}$ and $a_{i}$ will always denote an excess of loss and a proportional reinsurance limit respectively for the $i$-th risk; the symbol $\theta_{i}$ will be used to denote a reinsurance limit without specifying the type of reinsurance.

The premium charged by the reinsurer in respect of the $i$-th risk is $P_{i}\left(\theta_{i}\right)$ where $\theta_{i}$ is either $M_{i}$ or $a_{i}$. We denote by $F_{i}\left(\cdot, \theta_{i}\right)$ and $G_{i}\left(\cdot, \theta_{i}\right)$ the distribution function and moment generating function respectively of the net amount paid by the insurer in respect of a single claim arising from the $i$-th risk given a reinsurance limit $\theta_{i}$. For example

$$
G_{i}\left(t, M_{i}\right)=\int_{0}^{M_{i}} e^{t x} d F_{i}(x)+\exp \left\{t M_{i}\right\} \cdot\left(1-F_{i}\left(M_{i}\right)\right)
$$

Strictly speaking the notation $P_{i}\left(\theta_{i}\right), F_{i}\left(\cdot, \theta_{i}\right)$ and $G_{i}\left(\cdot, \theta_{i}\right)$ is not well defined since, for example, $P_{i}(1)$ has two different meanings depending on whether we are discussing excess of loss or proportional reinsurance. This sort of confusion should not arise in the rest of this paper.

\section{DISCUSSION}

The variables of our model are the $n$ reinsurance limits $\left(\theta_{1}, \theta_{2}, \ldots, \theta_{n}\right)$, where this vector is either $\left(M_{1}, M_{2}, \ldots, M_{n}\right)$ or $\left(a_{1}, a_{2}, \ldots, a_{n}\right)$; note that we do not consider a mixture of excess of loss and proportional reinsurance. We are going to investigate the effect on the "riskiness" of the insurer's portfolio of varying $\left(\theta_{1}, \theta_{2}, \ldots, \theta_{n}\right)$ and to do this we need a measure of the portfolio's degree of risk as a function of $\left(\theta_{1}, \theta_{2}, \ldots, \theta_{n}\right)$. Our candidate for this role is what we term the insurer's "net insolvency constant". The insurer's net insolvency constant, $R \equiv R\left(\theta_{1}, \theta_{2}, \ldots, \theta_{n}\right)$, is defined to be the unique positive root of

$$
\sum_{i=1}^{n} p_{i}+R .\left\{P-\sum_{i=1}^{n} P_{i}\left(\theta_{i}\right)\right\}-\sum_{i=1}^{n} p_{i} G_{i}\left(R, \theta_{i}\right)=0
$$

if this exists, or zero otherwise. It can be seen that $R\left(\theta_{1}, \theta_{2}, \ldots, \theta_{n}\right)$ is just the insurer's insolvency constant (or adjustment coefficient) as defined, for example, in Beard, Pentikäinen and Pesonen (1977, p. 144) or Gerber (1979, p. 118), taking into account the net claims paid and net income received by the insurer. 
The conditions necessary and sufficient for the existence of the positive root of (4) are firstly

$$
P-\sum_{i=1}^{n} P_{i}\left(\theta_{i}\right)>\sum_{i=1}^{n} p_{i} \int_{0}^{\infty} x d F_{i}\left(x, \theta_{i}\right)
$$

i.e. the insurer's total net income should exceed his total net expected claims, and secondly that the moment generating functions $G_{i}\left(\cdot, \theta_{i}\right)$ should not behave badly (in particular, should not jump to $+\infty$ at an inconvenient point). We can assume without any practical loss of generality that these moment generating functions are sufficiently well behaved.

The motivation for considering the net insolvency constant as a risk measure is provided by Lundberg's inequality which tells us that if the reinsurance limits are set at $\left(\theta_{1}, \theta_{2}, \ldots, \theta_{n}\right)$ then the probability that the insurer's accumulated surplus on this portfolio will ever fall below $-U$ is bounded above by $\exp \left\{-\mathrm{U} . \mathrm{R}\left(\theta_{1}, \theta_{2}, \ldots, \theta_{n}\right)\right\}$ for any $\mathrm{U}>0$. A more obvious measure of the risk level of the portfolio would be the variance of the total net annual claims. See, for example, Bühlmann (1970, ch. 5) and Benktander (1974). Our opinion is that the net insolvency constant has two advantages as a risk measure when compared to the variance. The first is that it will generally take into account more moments of the net claims distribution than just the mean and variance. This can be seen by considering equation (12.20') of BEARD, Pentikäinen and Pesonen (1977) and will be illustrated numerically in the next section. The second is that the net insolvency constant depends not only on the net claims distribution but also on the net premium income. For example, it is easy to show that for a fixed net claims distribution the net insolvency constant is an increasing function of the net premium income. Note that, using Lundberg's inequality, the higher the insurer's net insolvency constant the less risky we consider his portfolio to be.

The starting point for this research was a paper by one of the present authors, WATERS (1979), in which he considered the effect on the insurer's net insolvency constant of varying the excess of loss reinsurance limits for the portfolio. He showed that, under certain conditions, $R\left(M_{1}, M_{2}, \ldots, M_{n}\right)$ is a uni-modal function of $\left(M_{1}, M_{2}, \ldots, M_{n}\right)$ and, in particular, that it has a unique and easily determinable maximum value. In the next section we consider excess of loss reinsurance. We state Waters' basic result for the sake of completeness and we follow this by giving an extension of this result in which we show how one of the more restrictive assumptions can be weakened without seriously weakening the conclusion of the result. We give some examples to illustrate our theoretical results and an example to indicate that when the technical assumptions necessary to prove the theoretical results are dropped the results still hold. In $\S 5$ we carry out a similar study of proportional reinsurance. The numerical results in $\S \S 4$ and 5 have all assumed $n=1$, i.e. 
we have considered just one risk, although the theoretical results hold for any $n \geqslant 1$. By assuming $n=1$ these examples have disguised an interesting feature of the theoretical results. This feature is discussed in $\S 6$. In $\S 7$ we use an example to study in detail the effect on the insurer of a quota share reinsurance arrangement. As usual, we measure this effect by means of the insurer's net insolvency constant. The model used in $\S 7$ is somewhat closer to reality than the rather idealized models of $\S \S 4,5$ and 6 since, for example, it takes account of the insurer's expenses and commission paid by the reinsurer to the insurer. In this sense, $\S 7$ can be regarded as an example of a "practical application" of the ideas of the previous sections. The insolvency constant is very closely linked to the notion of an exponential utility function (see GERBER (1979), p. 120) and it is tempting to think that a set of reinsurance limits which is optimal in that it maximizes the insurer's net insolvency constant might be related to the set of limits obtained by maximizing the insurer's expected utility with respect to an exponential utility function. This point is considered in the final section where it is shown that the two solutions are not the same but are similar in form.

\section{EXCESS OF LOSS REINSURANCE}

Throughout this section we restrict our attention to excess of loss reinsurance for our portfolio. We are interested in the behaviour of $R\left(M_{1}, M_{2}, \ldots, M_{n}\right)$ as a function of $\left(M_{1}, M_{2}, \ldots, M_{n}\right)$ and our basic theoretical result is as follows:

Result 1

We make the following additional assumptions:

(a) $d F_{i} / d x$ exists and is continuous everywhere

(b) $P_{i}\left(M_{i}\right)=\left(1+\alpha_{i}\right) \cdot \int_{M_{i}}^{\infty}\left(x-M_{i}\right) d F_{i}(x)$ for some $\alpha_{i}>0$.

(c) $\sum_{i=1}^{n} P_{i}(0)>P$.

Then $(i)$ there exists a unique set of points $\left(\hat{M}_{1}, \hat{M}_{2}, \ldots, \hat{M}_{n}\right)$ such that

$$
R\left(\hat{M}_{1}, \hat{M}_{2}, \ldots, \hat{M}_{n}\right)=\hat{M}_{1}^{-1} \log \left(1+\alpha_{1}\right)=\ldots=\hat{M}_{n}^{-1} \log \left(1+\alpha_{n}\right)
$$

(ii) for any set of points $\left(M_{1}, M_{2}, \ldots, M_{n}\right)$ we have

$$
R\left(M_{1}, M_{2}, \ldots, M_{n}\right) \leqslant R\left(\hat{M}_{1}, \hat{M}_{2}, \ldots, \hat{M}_{n}\right)
$$

(iii) if we impose the extra conditions that for $i=1,2, \ldots, n$ we have $F_{i}(x)<1$ for any $x<\infty$ then (ii) can be improved to

$$
R\left(M_{1}, M_{2}, \ldots, M_{n}\right)<R\left(\hat{M}_{1}, \hat{M}_{2}, \ldots, \hat{M}_{n}\right)
$$


for any set of points $\left(M_{1}, M_{2}, \ldots, M_{n}\right) \neq\left(\hat{M}_{1}, \hat{M}_{2}, \ldots, \hat{M}_{n}\right)$ and $R\left(M_{1}, M_{2}\right.$, $\left.\ldots, M_{n}\right)$ is a uni-modal function.

\section{Proof}

A proof of this result can be found in Waters (1979).

\section{Comments on Result 1}

Assumption (b) above requires the reinsurer to use the expected value principle to calculate his premiums (with the possibility of a different loading factor for each risk). This, together with assumption (a) are technicalities that do not seem to be necessary to ensure that $R\left(M_{1}, M_{2}, \ldots, M_{n}\right)$ is a uni-modal function. The crucial assumption is (c) which requires that if the reinsurer takes over the whole of each risk, his total premium shall exceed the total premium originally charged by the insurer. This does not appear to be a restrictive assumption and is clearly necessary in this paper since without it we would have $R(0,0, \ldots, 0)=+\infty$ so that by reinsuring the whole portfolio the insurer has zero probability of ruin, a result that is obvious from general considerations. The maximum value of the insurer's net insolvency constant and hence the values of $\hat{M}_{i}$ for $i=1,2, \ldots, n$, can easily be found as can be seen from Waters (1979, Lemma 4).

We would like to be able to show that the main conclusion of the above result, that $R\left(M_{1}, M_{2}, \ldots, M_{n}\right)$ is a uni-modal function, still holds without having to make any restrictive assumptions, in particular (a) and (b) above. A first step in this direction is the following result in which we replace assumption (a) above by a much weaker assumption.

\section{Result 2}

The assumptions are as for Result 1 except that we replace (a) by

$\left(a^{\prime}\right)$ for each $i, i=1,2, \ldots, n$, we assume there exists a sequence of continuous probability density functions which converge pointwise to $d F_{i} / d x$.

Then conclusions (i) and (ii) of Result 1 still hold.

\section{Proof:}

The proof is by an essentially straightforward limiting argument but the details are rather messy and so the proof is omitted. The interested reader is referred to ANDREADAKIS (1980).

\section{Examples}

Figure 1 illustrates the ideas of this section. We consider the case $n=1$, i.e. we consider a portfolio consisting of a single risk, and we have plotted $R(M)$ against $M$ under three different assumptions. Note that we have given 


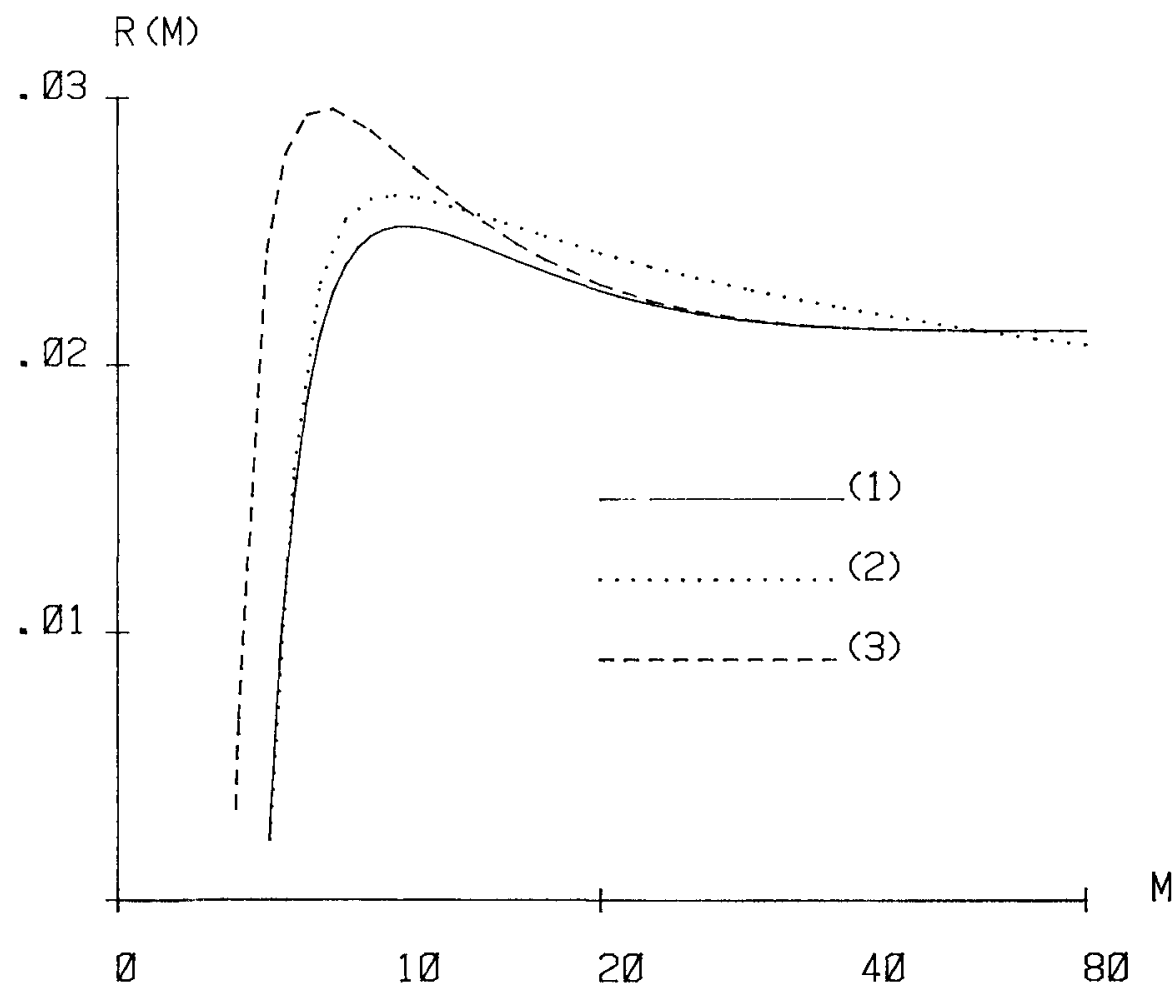

Fig. I

the $M$-axis an exponential scale so that all the interesting features of the graphs can be seen clearly. Each graph in Figure 1 uses one of the following two distribution functions to describe the size of a single claim. The first is a two parameter exponential distribution specified by

$$
d F / d x=0.2 \exp \{-0.2(x-5)\} \text { for } x \geqslant 5,=0 \quad \text { for } x<5
$$

and the second is a truncated Pareto distribution specified by

$$
\begin{aligned}
d F / d x & =3 x^{-4} /\left(6.7^{-3}-93 \cdot 3^{-3}\right) \quad \text { for } 6.7 x \leqslant 93.3 \\
& =0 \quad \text { for } x<6.7 \text { and } x>93.3 .
\end{aligned}
$$

Each of these two distributions has mean 10 and variance 25 . The reason for choosing these distributions to illustrate our results is that we can think of them as being "well behaved" and "badly behaved" respectively. See BERLINER (1977). In fact the Pareto behaves so badly we have to truncate it to ensure its moment generating function exists. For all three examples illustrated in Figure 1 the insurer's total annual premium is

$$
P=(1 \cdot 15) \cdot 10 . p
$$

where, as will become clear, the Poisson parameter $p$ need not be specified. 
For graph 1 we have used the two parameter exponential claims distribution and have assumed the reinsurance premium is calculated using the expected value principle with a $30 \%$ loading, i.e.

$$
P(M)=(1 \cdot 3) \cdot p \cdot \int_{M}^{\infty}(x-M) d F(x)
$$

It can be seen that graph 1 has the uni modal shape we expect. The maximum value of $R(M)$ is 0.0252 and this occurs at $\hat{M}=10.41$ (Note that $\hat{M} \cdot R(\hat{M})=$ $\log (1.3)$ as expected). This compares with the value $R(M=\infty)=0.0213$ if there is no reinsurance.

For graph 2 we have used the truncated Pareto claims distribution and the same formula as before for the calculation of the reinsurance premium, i.e. (12). The first point to note about this graph is its similarity in shape to the first one despite the different characteristics of the claims distributions. For this graph $R(M)$ achieves a maximum value of 0.0264 at the point $\hat{M}=9.95$ $\left(=0.0264^{-1} \cdot \log (1.3)\right)$. It is interesting to note that in this case the value of $R(M=\infty)$ is 0.0207 compared to 0.0213 for the previous graph and the values of the coefficients of skewness for the two claims distributions involved are 5.01 and 2.00 respectively. This is an illustration of the point made about the insolvency constant in $\S 3$.

For graph 3 we do not assume the expected value principle for the calculation of the reinsurance premium. We use the two parameter exponential distribution and assume the reinsurance premium is calculated by the exponential principle with parameter 0.0383. See GERBER (1979, p. 68). i.e.

$$
P(M)=\frac{P}{A}\left[\int_{M}^{\infty} \exp \{A(x-M)\} d F(x)+F(M)-1\right] \text { where } A=0.0383
$$

The parameter $A$ has been chosen so that $P(M=0)=(1 \cdot 3) \cdot 10 \cdot p$ as in (12). Again, we can see that the graph has the same general shape as the previous two examples although its peak has been moved upwards and leftwards for reasons that are not hard to explain. The maximum value of $R(M)$ is 0.0296 and this occurs at $M=7 \cdot 17$.

More examples can be found in Andreadakis (1980).

\section{Proportional Reinsurance}

We now turn our attention to proportional reinsurance and carry out a study similar to that of the previous section. In this case the basic theoretical result is as follows: 
Result 3

We make the following assumptions in addition to those we have already made about $P, F_{i}$ and $G_{i}$ in sections 2 and 3 :

(a) $P_{i}\left(a_{i}\right)=p_{t}\left\{\int_{0}^{\infty} \exp \left[A_{i}\left(1-a_{i}\right) x\right] d F_{i}(x)-1\right\} / A_{i}$

for some $A_{i}>0 \quad i \quad 1,2, \ldots, n$

(b) $\sum_{i=1}^{n} P_{i}(0)>P$.

Then (i) there is a unique set of points $\left(\hat{a}_{1}, \hat{a}_{2}, \ldots, \hat{a}_{n}\right)$ such that

$$
R\left(\hat{a}_{1}, \hat{a}_{2}, \ldots, \hat{a}_{n}\right)=A_{1} \frac{\left(1-\hat{a}_{i}\right)}{\hat{a}_{i}}=\ldots=A_{n} \frac{\left(1-\hat{a}_{n}\right)}{\hat{a}_{n}}
$$

and such that $0 \leqslant \hat{a}_{i} \leqslant 1$ for $i=1,2, \ldots, n$

(ii) for any set of points $\left(a_{1}, a_{2}, \ldots, a_{n}\right) \neq\left(\hat{a}_{1}, \hat{a}_{2}, \ldots, \hat{a}_{n}\right)$ we have

$$
R\left(a_{1}, a_{2}, \ldots, a_{n}\right)<R\left(\hat{a}_{1}, \hat{a}_{2}, \ldots, \hat{a}_{n}\right)
$$

(iii) $R$ is a uni-modal function of $\left(a_{1}, a_{2}, \ldots, a_{n}\right)$.

\section{Proof}

The proof is essentially simple: one substitutes the assumed form of $P_{i}\left(a_{i}\right)$ into equation (4), differentiates (4) with respect to $a_{1}, i=1,2, \ldots, n$, puts these partial derivatives equal to zero and solves the resulting set of equations for $a_{i}$. There are, however, three complications to be overcome. The first is to show that $R\left(a_{1}, a_{2}, \ldots, a_{n}\right)$ is a sufficiently smooth function-this requires an application of the Implicit Function Theorem. The second is to show that there is a unique set of points $\left(\hat{a}_{1}, \hat{a}_{2}, \ldots, \hat{a}_{n}\right)$ which satisfy (14). The third is to show that the point $\left(\hat{a}_{1}, \hat{a}_{2}, \ldots, \hat{a}_{n}\right)$ is a global maximum and not just a local maximum for the function $R$. The proofs of these points are somewhat messy and are omitted. The interested reader is referred to ANDREADAKIS (1980).

\section{Comments on result 3}

Assumption (a) above requires the reinsurance premium for the $i$-th risk to be calculated using the exponential principle with parameter $A_{i}$. This is the counterpart of assumption (b) in the statement of Result 1. From numerical 
examples it seems that this condition is not necessary to ensure that $R$ is a uni-modal function. One such example is given below. The crucial assumption is (b) which is the same as, and plays the same role as, assumption (c) in Result 1. The essence of the result is that, provided the insurer cannot reinsure the whole portfolio and still make a profit, his net insolvency constant is a uni-modal function of the proportional reinsurance limits. In particular, if the reinsurance premiums are calculated according to the exponential principle, the maximum value of the net insolvency constant can easily be found. See ANDREADAKIS (1980, Lemma 3.2.3). Hence the values $\hat{a}_{i}$ can easily be found.

\section{Examples}

As in the previous section we illustrate our ideas by considering the case $n=1$ and plotting $R(a)$ against $a$ under three different assumptions. These three graphs are shown in Figure 2. In each case the claims distribution is either the two parameter exponential specified by (9) or the truncated Pareto specified by (10). The insurer's total premium is, as before, given by (11).

For graph 1 we have used the two parameter exponential claims distribution and we have assumed the reinsurance principle is calculated using the exponential principle with parameter 0.0383 , so that $P(a=0)=(1 \cdot 3) .10 . p$. As predicted by Result 2 this graph has a uni-modal shape. The maximum value of $R(a)$ is 0.0480 which occurs at $\hat{a}=0.444$. (Note that $0.444=0.0383 /[0.0383+0.0480]$ to a reasonable degree of accuracy). The value of $R(a=1)$ is, as is known from the previous section, 0.0213 .

For graph 2 in Figure 2 we have used the truncated Pareto claims distribution and assumed the reinsurance premium is calculated using the exponential principle with parameter 0.0360 so that, again, $P(a=0)=(1 \cdot 3) .10 . p$. This graph is very similar in shape to graph 1 . (See the comments on graphs 1 and 2 in Figure 1.) The maximum value of $R(a)$ is 0.0487 which occurs at $\hat{a}=0.425$ $(=0.0360 /[0.0360+0.0487])$.

For graph 3 in Figure 2 we have used the two parameter exponential claims distribution but we have assumed this time that the reinsurance premium is calculated by the expected value principle with a $30 \%$ loading. This results in a very different, but still uni-modal, shape for $R(a)$. The maximum value of $R(a)$ is 0.0214 which occurs at $\hat{a}=0.947$.

Although it would be rash to draw too many general conclusions from the few, somewhat artificial, examples we have presented so far, it does seem clear from both Figures 1 and 2 that for fixed mean and variance of the claims amount distribution the shape of the graph of $R$ depends more on the loadings included in the insurer's and reinsurer's premiums than on the particular form of the claims amount distribution. For further examples see ANDrEadakis (1980). 


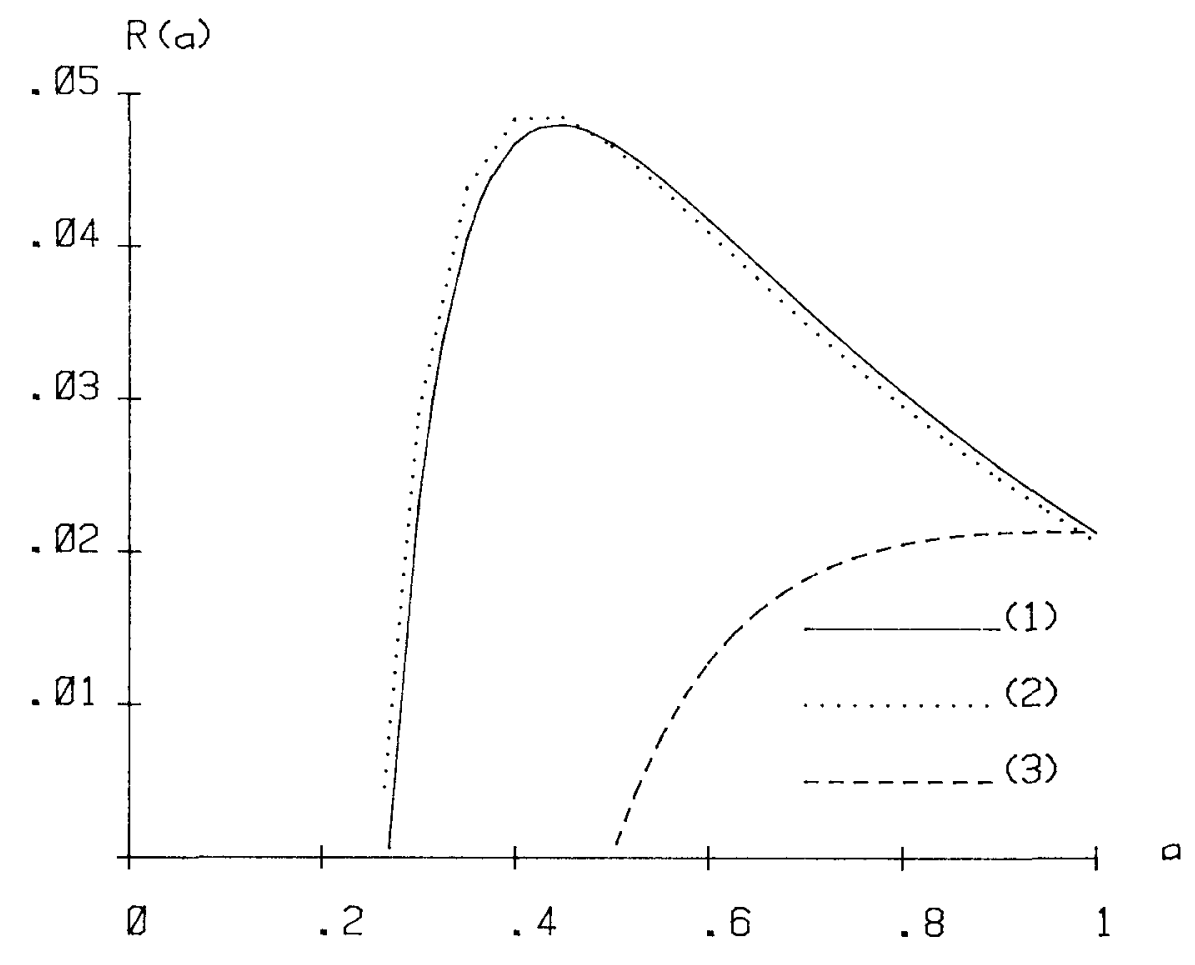

Fig. 2

6. TWO OR MORE RISKS

The theoretical results we have discussed so far are applicabie for any value of $n$, i.e. for any finite number of risks, but our numerical results have all assumed $n=1$. There is an interesting point concerning our theoretical results that has not been brought out by our examples and it is this point we shall discuss in this section

To illustrate our point we return to the assumptions of Result 2, i.e. we consider excess of loss reinsurance and we assume the reinsurance premiums are calculated using the expected value principle. We also assume, for the sake of clarity, that $n=2$ but the following remarks apply for any $n>1$. Let $\Pi_{1}$ and $\Pi_{2}$ denote the insurer's total annual premiums for the two risks so that $P=\Pi_{1}+\Pi_{2}$. We start by considering each risk separately. For the $i$-th risk the insurer's net insolvency constant, $R\left(M_{i}\right)$, is the unique positive root of

$$
H_{i}(R) \equiv p_{i}+R \cdot\left[\Pi_{i}-P_{i}\left(M_{i}\right)\right]-p_{i} G_{i}\left(R, M_{i}\right)=0 \quad i=1,2 .
$$

if this exists or zero otherwise. We know from Result 2 that $R\left(M_{i}\right)$ achieves its maximum value at a point $\hat{M}_{i}$ where $\hat{M}_{i}=R\left(\hat{M}_{i}\right)^{-1} \cdot \log \left(1+\alpha_{i}\right)$. 
We now consider the two risks together. The insurer's net insolvency constant, $R\left(M_{1}, M_{2}\right)$, is now the unique positive root of

$$
H_{1}(R)+H_{2}(R)=0
$$

if this exists or zero otherwise. Again using Result 2 we can say that $R\left(M_{1}, M_{2}\right)$ achieves its maximum value at a point $\left(\hat{\bar{M}}_{1}, \hat{\bar{M}}_{2}\right)$ where $\hat{\bar{M}}_{i}=R\left(\hat{M}_{1}, \hat{\bar{M}}_{2}\right) \cdot \log$ $\left(1+\alpha_{i}\right)$ for $i=1,2$. The problem is that $R\left(\hat{M}_{1}, \hat{M}_{2}\right)$ may not be equal to $R\left(\hat{M}_{1}\right)$, say, since the former depends on the value of $\Pi_{2}$ whereas the latter does not. Hence it is possible that $\hat{M}_{1} \neq \hat{M}_{1}$ and/or $\hat{M}_{2} \neq \hat{M}_{2}$ (It can be seen from Result 3 that a similar problem arises in proportional reinsurance). In other words, if we have more than one risk and if we regard as optimal a set of retention limits that maximizes the insurer's net insolvency constant, then what is optimal when each risk is considered individually may not be optimal when the risks are considered together. Numerical examples to illustrate this point can be found in ANDREADAKIs (1980).

The rest of this section is devoted to proving some simple results that shed some light on the above remarks. For the sake of clarity we consider just two risks but, as before, the following results have obvious extentions to any $n>1$. We do not specify the type of reinsurance we are considering and we make no assumptions about the way the reinsurance premiums are calculated. For $i=1,2, R\left(\theta_{1}\right)$ is, as before, the insurer's net insolvency constant when the $i$-th risk is considered on its own. i.e. $R\left(\theta_{i}\right)$ is the unique positive root of

$$
K_{i}(R) \equiv p_{i}+R \cdot\left[\Pi_{i}-P_{i}\left(\theta_{i}\right)\right]-p_{i} \cdot G_{i}\left(R, \theta_{i}\right)=0
$$

if this exists or zero otherwise. $R\left(\theta_{1}, \theta_{2}\right)$ is, as before, the insurer's net insolvency constant when both risks are considered together. Then we have the following result.

\section{Result 4}

For fixed $\theta_{1}$ and $\theta_{2}$ we have

$$
\min \left\{R\left(\theta_{1}\right), R\left(\theta_{2}\right)\right\} \leqslant R\left(\theta_{1}, \theta_{2}\right) \leqslant \max \left\{R\left(\theta_{1}\right), R\left(\theta_{2}\right)\right\}
$$

\section{Proof}

The proof is very simple. We can assume that o $<R\left(\theta_{1}\right) \leqslant R\left(\theta_{2}\right)$. By considering $K_{i}(\mathrm{o}), K_{i}^{\prime}(\mathrm{o}), K_{i}^{\prime \prime}(R)$ and $\operatorname{Lim} K_{i}(R)$ it can be seen that $R \rightarrow \infty$

$$
K_{i}(R)>0 \text { if } 0<R<R\left(\theta_{i}\right), K_{i}(R)<0 \text { if } R>R\left(\theta_{i}\right), i=1,2, .
$$

$R\left(\theta_{1}, \theta_{2}\right)$ is the unique positive root of $K_{1}(R)+K_{2}(R)=0$. 
From (20) we have that

$$
K_{1}\left(R\left(\theta_{1}\right)\right)+K_{2}\left(R\left(\theta_{1}\right)\right)=K_{2}\left(R\left(\theta_{1}\right) \geqslant 0\right.
$$

since, by assumption, $R\left(\theta_{2}\right) \geqslant R\left(\theta_{1}\right)$. Similarly

$$
K_{1}\left(R\left(\theta_{2}\right)\right)+K_{2}\left(R\left(\theta_{2}\right)\right)=K_{1}\left(R\left(\theta_{2}\right)\right) \leqslant 0
$$

(19) then follows immediately.

The relevance of Result 4 to the remarks earlier in this section can be seen in the following corollary.

\section{Result 5}

If $R\left(\theta_{1}\right)$ achieves its maximum value at $\theta_{i}=\hat{\theta}_{i}, i=1,2$, and if $R\left(\theta_{1}, \theta_{2}\right)$ achieves its maximum value at $\left(\theta_{1}, \theta_{2}\right)=\left(\hat{\bar{\theta}}_{1}, \hat{\hat{\theta}}_{2}\right)$ then

$$
\min \left\{R\left(\hat{\theta}_{1}\right), R\left(\hat{\theta}_{2}\right)\right\} \leqslant R\left(\hat{\theta}_{1}, \hat{\theta}_{2}\right) \leqslant \max \left\{R\left(\hat{\theta}_{1}\right), R\left(\hat{\theta}_{2}\right)\right\}
$$

\section{Proof}

From Result 4 and the definition of $\left(\hat{\hat{\theta}}_{1}, \hat{\hat{\theta}}_{2}\right)$ we have

$$
\min \left\{R\left(\hat{\theta}_{1}\right), R\left(\hat{\theta}_{2}\right)\right\} \leqslant R\left(\hat{\theta}_{1}, \hat{\theta}_{2}\right) \leqslant R\left(\hat{\hat{\theta}}_{\mathbf{1}} \hat{\hat{\theta}}_{2}\right)
$$

This proves the first part of (23). Similarly

$$
R\left(\hat{\hat{\theta}}_{1}, \hat{\theta}_{2}\right) \leqslant \max \left\{R\left(\hat{\theta}_{1}\right), R\left(\hat{\theta}_{2}\right)\right\} \leqslant \max \left\{R\left(\hat{\theta}_{1}\right), R\left(\hat{\theta}_{2}\right)\right\}
$$

\section{A MORE PRACTICAL EXAMPLE}

The examples we have used to illustrate our ideas in $\S \S 4$ and 5 have been somewhat artificial. What we are going to do in this section is to use the same ideas in, what we hope is, a more practical setting. We are going to study an example involving quota share reinsurance. This example has its origins in Carter (1979, p. 105).

Our example has the following specifications:

(a) We consider a single risk.

(b) The total annual claims from this risk has a compound Poisson distribution with 100 claims expected each year and with each claim having a gamma distribution

$$
d F / d x=x^{4 \cdot 5} \cdot \exp \left\{-x \cdot 5 \cdot 10^{-4}\right\} \cdot /\left[\Gamma(5 \cdot 5) \cdot\left(2 \cdot 10^{3}\right)^{5 \cdot 5}\right] \text { for } x \geqslant 0 .
$$

which has mean 11,000 and standard deviation 4,690.

(c) The insurer's gross annual premium is $P=2.10^{6}$ of which a proportion $e=0.35$ is required to cover the insurer's expenses irrespective of 
whether he arranges reinsurance or of the retention limit for reinsurance.

(d) The insurer arranges quota share reinsurance for this risk; he retains a proportion a of each claim and the reinsurer pays a proportion (1-a).

(e) The reinsurance premium is $P .(1-a)=2.10^{6} .(1-a)$ of which a proportion $c=0.33$ is passed back to the insurer as a commission payment.

Hence the insurer's net income, after paying expenses and the reinsurance premium is $P[c-e+a(1-c)]$. We want to investigate the effect on the risk level of the insurer's portfolio of varying the retained proportion $a$ and we do this, as usual, by calculating the insurer's net insolvency constant as a function of $a$, i.e. looking at the unique positive root, $R=R(a)$, of

$$
p+R \cdot P[c-e+a(1-c)]-p \int_{-\infty}^{\infty} \exp \{R \cdot a \cdot x\} d F(x)=0
$$

where $p, P, c, e$ and $F$ are as specified above. Note that by taking $c<e$ we have prevented the insurer from being able to reinsure the whole of the risk and making a profit. This is analogous to assumptions (c) and (b) in Results 1 and 3 respectively. The first point to emerge from a study of $R(a)$ as a function of $a$ is that for $a \leqslant 0 \cdot 1666$ the insurer's net income is less than his net expected claims so that $R(a)=0$. The next point is that for $0.1666 \leqslant a \leqslant 1, R(a)$ is a quite sharply peaked uni-modal curve similar to graphs 1 and 2 in Figure 2. The numerical points of interest are that the maximum value of $R(a)$ is $4 \cdot 66.10^{-5}$ and this occurs at $a=0.32$. If there is no reinsurance the insurer's net insolvency constant, $R(a=1)$, is $2 \cdot 46 \cdot 10^{-5}$.

One conclusion that could be drawn from the above example is that reinsurance is worthwhile for the insurer since whatever his initial reserves, $U$, the upper bound for his probability of exhausting these reserves can be reduced from $\exp \left\{-2 \cdot 46 \cdot 10^{-5} . U\right\}$ to as low as $\exp \left\{-4 \cdot 66 \cdot 10^{-5}\right.$. $\left.U\right\}$. It is of obvious interest to ask the question "Given $p, P, e$ and $F$, for what range of values of $c$ is reinsurance worthwhile for the insurer ?" In other words "How high must the commission rate be for reinsurance to be worthwhile?" The remainder of this section will be devoted to trying to provide an answer to this question.

Our model is as outlined earlier in this section and the symbols $p, P, c, e, F$ and $a$ have the same general meaning (although not necessarily the same numerical values) as before. We make two basic assumptions: the first is that $c<e$ (see the earlier remarks) and second is that

$$
P(1-e)>p \int_{0}^{\infty} x d F(x)
$$

which corresponds to assumption (1). The insurer's net insolvency constant is defined as the unique positive root of $(27)$ if this exists or zero otherwise. 
It is easy to see there exists some $L \in(0,1)$ such that $R(a)$ is zero if and only if a $\in[0, L]$. ( $L$ is the value of a for which the insurer's net premium equals his net expected claims, which is 0.1666 in the numerical example.) We can now prove the following result.

\section{Result 6}

A necessary and sufficient condition for there to be a point $a^{\prime} \leqslant[0,1]$ such that $R\left(a^{\prime}\right)>R(a=1)$ is that

$$
C>1-p\left[\int_{0}^{\infty} x \cdot \exp \{R(a=1) \cdot x\} d F(x)\right] / P
$$

and if this condition holds then $R(a)$ is a uni-modal function with a unique maximum at $\hat{a} \in(L, 1)$. If this condition does not hold then $R(a)$ is a monotonic non-decreasing function on $[L, 1]$.

\section{Proof}

The first step in the proof is to show that $R(a)$ is a continuous function of $a$ $\in[L, 1]$ and differentiable on $(L, 1)$ a sufficient number of times. This is a standard application of the Implicit Function Theorem and the details are omitted. If we differentiate (27) with respect to $a$ then it can be seen that $\delta R / \delta a$ $=0$ if and only if either $R=0$, which is of little interest to us, or

$$
c=1-p\left[\int_{0}^{\infty} x \cdot \exp \{R(a) \cdot a \cdot x\} d F(x)\right] / P
$$

This is an implicit equation for $a$ and it is not yet clear how many roots there are, if any, in the interval $[L, 1]$. However, if we differentiate $(27)$ twice with respect to $a$, then assume $\delta R / \delta a=0$ and use (30) we can show that $\delta R / \delta a=$ o implies $\delta^{2} R / \delta a^{2}<0$. Hence, any turning point of $R(a)$ for $a \in[L, 1]$ must be a maximum. Since we know that $R(a)>0$ for $a \in(L, 1]$ this shows that $R(a)$ is either monotonically non-decreasing in $[L, 1]$ or is uni-modal with a unique maximum in $(L, 1)$. Which of these two shapes $R(a)$ has depends on the sign of the limit as $a \rightarrow 1-0$ of $\delta R / \delta a$; this will be positive or zero for the former and negative for the latter. Using (27) it can be shown that this limit is

$$
\begin{aligned}
& R^{2} \cdot\left[p \int_{0}^{\infty} \exp \{R(a=1) \cdot x\} d F(x)-P(1-c)\right] / \\
& I\left[p \int_{0}^{\infty}[1-\exp \{R(a=1) \cdot x\}-x \cdot R(a=1) \cdot \exp \{R(a=1) \cdot x\}] d F(x)\right]
\end{aligned}
$$

and since the denominator is always negative, (29) follows. 


\section{Comment on Result 6}

If we return to the numerical example discussed earlier in this section and we evaluate the right hand side of $(29)$ it will be found to be $0 \cdot 235$. The conclusion of Result 6 is that reinsurance of this risk on the terms assumed earlier is only worthwhile to the insurer, in the sense that it can be used to reduce the level of risk, if the commission rate is greater than $23 \frac{1}{2} \%$, as it was in our example.

\section{Maximizing Expected Utility}

Throughout this paper our aim has been to study the effect on the insurer of varying the levels of reinsurance on his portfolio and we have measured this effect by means of his net insolvency constant. To take a narrower view, we have shown that under certain circumstances the net insolvency constant is a uni-modal function with a unique maximum and we can regard any set of reinsurance limits which maximizes this function as optimal. Insolvency constants are closely connected with utility theory with respect to an exponential utility function (see GERBER (1979)) and in this section we want to consider briefly what connection, if any, exists between a set of reinsurance limits that is optimal in the sense just discussed and a set of reinsurance limits that is optimal in the sense that it maximizes the insurer's expected utility of wealth with respect to an exponential utility function. We start by doing this in the case of excess of loss reinsurance.

\section{Result 7}

The assumptions and notation are as in Result 2 and in addition, to avoid unnecessary complications, we assume $F_{i}(x)<1$ for $x<\infty, i=1,2, \ldots, n$. Then the insurer's expected utility of wealth at the end of the year is maximized with respect to the exponential utility function.

$$
u(x)=[1-\exp \{-\theta \cdot x\}] / \theta \quad \text { where } \theta>0
$$

if the excess of loss reinsurance limits are given by

$$
M_{i}=\theta^{-1} \cdot \log \left(1+\alpha_{i}\right) \quad i=1,2, \ldots, n
$$

Proof:

The proof of this result is straightforward. The quantity to be maximized is

$$
\text { (34) } \begin{aligned}
E\left(M_{1}, M_{2}, \ldots, M_{n}\right)=\int_{0}^{\infty} \theta^{-1} \cdot\left[1-\exp \left\{-\theta \cdot\left(W+P-\sum_{i=1}^{n}\left(1+\alpha_{i}\right) p_{i} \int_{M_{i}}^{\infty} x d F_{i}(x)\right.\right.\right. \\
\left.\left.\left.+\sum_{i=1}^{n}\left(1+\alpha_{i}\right) \cdot p_{i} \cdot M_{i} \cdot\left(1-F_{i}\left(M_{i}\right)\right)-y\right)\right\}\right] d B\left(y, M_{1}, M_{2}, \ldots, M_{n}\right)
\end{aligned}
$$


where $W$ is the insurer's initial wealth and $B\left(., M_{1}, M_{2}, \ldots, M_{n}\right)$ is the distribution function of the insurer's total net annual claims given the reinsurance limits $\left(M_{1}, M_{2}, \ldots, M_{n}\right)$. Using standard properties of compound Poisson distributions we can show that

$$
\begin{gathered}
E\left(M_{1}, M_{2}, \ldots, M_{n}\right)=\theta^{-1}-\theta^{-1} \cdot \exp \left\{-\theta\left[W+P-\sum_{i-1}^{n} p_{i}\left(1+\alpha_{i}\right) \int_{M_{i}}^{\infty}\left(x-M_{i}\right) d F_{i}(x)\right]\right\} . \\
. \exp \left\{\sum_{i=1}^{n}\left[p_{i} \int_{0}^{M_{i}} \exp \{\theta \cdot y\} d F_{i}(y)+P_{i} \cdot \exp \left\{\theta \cdot M_{i}\right\} \cdot\left(1-F_{i}\left(M_{i}\right)\right)-\sum_{i=1}^{n} p_{i}\right\}\right.
\end{gathered}
$$

The result follows by differentiating (35) with respect to $M_{i}$.

The corresponding result for proportional reinsurance is:

\section{Result 8}

The assumptions and notation are the same as for Result 3 . Then the insurer's expected utility of wealth at the end of the year is maximized with respect to the exponential utility function (32) if the proportional reinsurance limits are given by

$$
a_{i}=A_{i} /\left(\theta+A_{i}\right) \quad i=1,2, \ldots, n
$$

\section{Proof}

The proof is similar to that of the previous result.

\section{Comments on Results 7 and 8}

The interesting point about the above two results is the similarity between (33) and (6) on the one hand and between (36) and (14) on the other. In each case we have a given parameter $\theta$ replacing the maximum value of the net insolvency constant. However, this important difference does show why our earlier results are not equivalent to maximizing the insurer's expected utility of wealth. It is also interesting to note that Result 8 can be seen as a consequence of a result of GERBER $(1974$, p. 221).

\section{REFERENCES}

Andreadakis, M. (1980). The Insurer's Net Insolvency Constant and the Problem of Optimal Reinsurance Limits. M. Sc. Thesis presented to Heriot-Watt University, Edinburgh.

Beard, R. E., Pentikäinen, T. and Pesonen, E. (1977). Risk Theory. Chapman and Hall, ILondon.

Benktander, G. (1974). A Note on Optimal Reinsurance. Astin Bulletin, 8, $154-163$.

Berliner, B. (1977). Analytic Representation of Claim Distributions. Giro Bulletin, $15,26-32$.

Bühlmann, H. (1970). Mathematical Methods in Risk Theory. Springer, New York.

Carter, R. I. (1979), Reinsurance. Kluwer, London. 
Gerber, H. U. (1974). On Additive Premium Calculation Principles. Astin Bulletin, 7, 205-2 16.

Gerber, H. U. (1979). An Introduction to Mathematical Risk Theory. S. S. Huebner Foundation, Philadelphia.

Waters, H. R. (1979). Excess of Loss Reinsurance Limits. Scandinavian Actuarial Journal, 1979, 37-43. 